\title{
Uptake of cobalamin and markers of cobalamin status: a longitudinal study of healthy pregnant women
}

\author{
Eva Greibe ${ }^{a}$ **, Birgitte Horst Andreasena, Dorte L. \\ Lildballe, Anne L. Morkbak, Anne-Mette Hvas and \\ Ebba Nexo \\ Department of Clinical Biochemistry, Aarhus University \\ Hospital, Aarhus, Denmark
}

\begin{abstract}
Background: Currently, it is unknown whether the decline in plasma cobalamin observed during pregnancy is caused by malabsorption of the vitamin. This study examined cobalamin absorption and markers of cobalamin status during normal pregnancy.

Methods: Twenty-seven pregnant Danish women were examined at gestation weeks 13, 24 and 36. The absorption test CobaSorb was performed in all women implying measurement of holotranscobalamin or cyanocobalamin bound to transcobalamin before and after 2 days intake of $3 \times 9 \mu \mathrm{g}$ cobalamin. Serum cobalamin and the two cobalamin binding proteins transcobalamin and haptocorrin, including haptocorrin saturated with cobalamin or analogues, were measured, and so was plasma methylmalonic acid and homocysteine.

Results: No change in the uptake of cobalamin was observed throughout pregnancy. Serum cobalamin displayed a gradual decline during pregnancy $(\mathrm{p}<0.0001)$, while holotranscobalamin remained unchanged, despite an increase in total transcobalamin $(\mathrm{p}<0.0001)$. In accord with these results, total haptocorrin showed a decline from the 1st to 3rd trimester $(p=0.007)$ and cobalamin bound to haptocorrin declined $(p<0.0001)$. Interestingly, the amount of cobalamin analogues attached to haptocorrin remained unchanged. Methylmalonic acid $(p=0.002)$ and homocysteine $(p<0.0001)$ increased during pregnancy.

Conclusions: Cobalamin absorption remains unchanged during normal pregnancy, as judged by the CobaSorb test. No change was observed in the biological active holotranscobalamin during pregnancy. Thus, the pregnancy-related decline in cobalamin is caused by alternations in haptocorrinbound cobalamin. Surprisingly, no pregnancy-related change was observed in the amount of analogues attached to haptocorrin.
\end{abstract}

\footnotetext{
$\overline{{ }^{a}}$ Eva Greibe and Birgitte Horst Andreasen share first authorship. Both authors contributed equally to the work.

*Corresponding author: Eva Greibe, Department of Clinical Biochemistry, Aarhus University Hospital, Norrebrogade 44, building 9, 8000 Aarhus C, Denmark

Phone: +45 8949 3018, Fax: +45 8949 3060,

E-mail: evagreibe@gmail.com

Received February 28, 2011; accepted July 27, 2011;

previously published online August 30, 2011
}

Keywords: absorption; cobalamin; haptocorrin; holotranscobalamin; pregnancy; vitamin $\mathrm{B}_{12}$.

\section{Introduction}

Cobalamin (vitamin $\mathrm{B}_{12}$ ) is essential for one-carbon metabolism and cell division and important for normal fetal development $(1,2)$. The unborn child depends on the mother's ability to accumulate adequate amounts of cobalamin from the diet (3) and biochemical studies of cobalamin status during gestation suggests a pregnancy-related decrease in the amount of circulating cobalamin $(4,5)$. In the circulation, cobalamin consists of two fractions; the fraction bound to transcobalamin, holotranscobalamin (holoTC), that is available for the cells, and the fraction bound to haptocorrin, holohaptocorrin (holoHC), of which the function remains unknown. At present it is unknown if the decline in cobalamin reflects a change in absorption during pregnancy, an increased exploitation of cobalamin in the pregnant woman or a change in the cobalamin binding proteins in the plasma. Recent studies suggest that the decrease in cobalamin during pregnancy can be explained by a decrease in holoHC, rather than the biological active holoTC and propose that this decrease is caused by an increase in cobalamin analogues of no known biological function bound to haptocorrin (HC) (6).

Previous methods for evaluating intestinal cobalamin absorption relied on measurement of radioactively-labeled cobalamin after oral ingestion (7) and, therefore, these methods have not been considered suitable for use during pregnancy. The CobaSorb assay is a newly-developed method to estimate cobalamin absorption (8-10). The test takes advantage of measuring plasma levels of the active fraction of cobalamin available to the cells, namely cobalamin bound as holoTC, before and after intake of an oral test dose of the vitamin.

In the present study, we evaluated cobalamin absorption by use of the CobaSorb test and measured metabolic markers of cobalamin deficiency - including the binding of cobalamin and analogs to $\mathrm{HC} \mathrm{-} \mathrm{in} 27$ healthy pregnant women three times during gestation.

\section{Materials and methods}

\section{Participants and study design}

This longitudinal cohort study included 27 healthy Danish women (inclusion criteria age range 20-40 years) all pregnant in week 13 $(-1 /+3)$. The women were recruited at the first trimester antenatal ultrasound scan at the Department of Gynecology and Obstetrics, Aarhus University Hospital, Denmark, from October 2008 to 
February 2009. Exclusion criteria were cobalamin treatment within the past 5 years, infectious disease or chronic systemic disease.

Maternal anthropologic measures and data on intake of vitamins, medicine, dietary habits, parity and pregnancy-related complications were obtained. The gestational age was defined based on the last menstrual date and the ultrasound examination.

The pregnant women were examined three times; at pregnancy weeks $13(-1 /+3), 24( \pm 1)$ and $36( \pm 1)$. At each visit, non-fasting EDTA blood and whole blood samples were collected from each participant and used for analysis of markers of cobalamin status and related parameters (see below). This sample also served as the day 1 sample for the CobaSorb test (see below).

The study was approved by the Central Denmark Region Ethics Committee (project number 20080090) and was performed within the confines of the Helsinki Declaration. All participants gave their informed consent before inclusion in the study.

\section{CobaSorb and C-CobaSorb}

The CobaSorb tests were carried out as previously described (8-10). In brief, holoTC was measured from non-fasting blood samples collected before (day 1) and after (day 3) intake of three oral doses of 9- $\mu \mathrm{g}$ cyanocobalamin $(\mathrm{CNCbl})$ (Natur Drogeriet A/S, Denmark) for 2 days (day 1 and day 2).

The CNCbl-tablets were given with orange or apple juice and a snack (fruit, bread) and the participants were allowed to eat their usual non-vegetarian diet throughout the study. The women were asked to discontinue intake of vitamin supplements with cobalamin a week before each absorption test. This recommendation was based on earlier findings of no significant difference in holoTC between baseline and 6 days post-intake of three oral doses of $9-\mu \mathrm{g} \mathrm{CNCbl}$ (8). The women were allowed to continue intake of folic acid throughout the study.

Judged by the CobaSorb test, cobalamin is absorbed if holoTC increases more than $10 \mathrm{pmol} / \mathrm{L}$ and more than $22 \%$ on day $3 \mathrm{com}-$ pared with baseline (day 1) (10). In the present study, we classified the participants as absorbers if they met these criteria for at least two of the three examinations. We analyzed $\mathrm{CNCbl}$ bound to $\mathrm{TC}$ in all samples from participants that showed a baseline holoTC $>65 \mathrm{pmol} / \mathrm{L}$ and increased $<10 \mathrm{pmol} / \mathrm{L}$ in holoTC (and consequently far below 22\%) between baseline and day 3 (six participants). This version of the test has been named C-CobaSorb (11), Employing C-CobaSorb, cobalamin is absorbed if $\mathrm{CNCbl}$ on $\mathrm{TC}$ increases more than $6 \mathrm{pmol} / \mathrm{L}$ and more than three-fold on day 3 compared to baseline (11).

Fifty-two healthy Danish non-pregnant individuals that previously underwent the CobaSorb test served as controls. They all had a baseline holoTC $<65 \mathrm{pmol} / \mathrm{L}$ and were classified by the CobaSorb test as cobalamin absorbers (10).

\section{Biochemical measurements}

Total serum cobalamin was assayed on a Cobas 6000 (Roche, Japan) with a total imprecision of 5\% and an intra-assay imprecision of $2 \%$. Plasma methylmalonic acid (MMA) was measured by a slightly modified stable-isotope-dilution capillary gas chromatography mass spectrometry with a total imprecision of $9.4 \%$ and an intra-assay imprecision of $3.5 \%$. Plasma total homocysteine (tHcy) and plasma creatinine were both measured by the Vitros 5.1 FS (Ortho Clinical Diagnostics) with a total imprecision of 5.3\%-7.8\% and an intraassay imprecision of $1.2 \%-3.8 \%$ for tHcy; and a total imprecision of $3 \%-3.8 \%$ and an intra-assay imprecision of $0.8 \%-1.1 \%$ for creatinine. Blood hemoglobin and erythrocyte mean volume (EMV) were both assessed on the Sysmex XE2100 platform (Sysmex,
Japan) with a total imprecision of $2.1 \%-2.6 \%$ and an intra-assay imprecision of $0.5 \%-0.7 \%$ for hemoglobin; and a total imprecision of $1.2 \%$ and an intra-assay imprecision of $0.5 \%-0.7 \%$ for EMV.

TotalTC was measured by an in-house sandwich ELISA with a total imprecision of $4 \%-6 \%$ and an intra-assay imprecision of 3\% (12). HoloTC was measured by the TC-ELISA after removal of the apoTC with cobalamin-coated beads (13). The total imprecision was $8 \%$ (13) and the intra-assay imprecision was 4\% (14). ApoTC was calculated as totalTC minus holoTC. TotalHC was measured by an in-house sandwich ELISA with a total imprecision of 5\% and the intra-assay imprecision of $2 \%$ (15). HoloHC was measured by the HC-ELISA after removal of the apoHC with cobalamin-coated magnetic beads as described previously for measurements of holoTC (13). The total imprecision was 5\% and the intra-assay imprecision was $2 \%$. ApoHC was calculated as totalHC minus holoHC. Measurement of analogs on $\mathrm{HC}$ (analog-HC) and cobalamins on $\mathrm{HC}$ (Cbl-HC) was performed as previously described (16). In brief, HC was immunoprecipitated with anti-HC coated magnetic beads and total corrinoids were released by proteolytic degradation. ApoTC (measurement of cobalamin) or apoHC (measurement of total corrinoids) were added to aliquots of the sample. The amount of saturated TC and $\mathrm{HC}$ was determined by ELISA after removal of the apo-proteins as described above. For the cobalamin assay, the total imprecision values were $8 \%-13 \%$ and the intra-assay imprecision values were $6 \%-11 \%$ (16). For the corrinoids assay, the total imprecision values were $8 \%-10 \%$ and the intra-assay imprecision values were $5 \%-8 \%$ (16). Analog-HC was calculated as the amount of corrinoids bound to $\mathrm{HC}$ (corr-HC) minus the amount of Cbl-HC. Measurement of $\mathrm{CNCbl}$ attached to $\mathrm{TC}$ was performed as described previously (11). In brief, TC was immunoprecipitated from serum and cobalamin was released by protease treatment, and $\mathrm{CNCbl}$ was isolated by HPLC and subsequently quantified with ELISA (total intra-assay imprecision 7\%-9\%) (11).

\section{Statistical analysis}

Logarithmic transformation was used to obtain normal distribution; except for tHcy-data, that were already normally distributed; and for EMV-data where normal distribution could not be achieved. EMV was tested by the non-parametric Wilcoxon signed rank test between weeks 13 and 24; and between weeks 24 and 36. Changes in medians of the measured biochemical markers were tested by the Hotellings T-square test for multivariate analyses, which is comparable to performing two-paired t-tests (week 13 vs. week 24 and week 24 vs. week 36), which again is the same as testing week $13=$ week $24=$ week 36 . p-Values $<0.05$ were regarded as statistically significant. In this regard, the Bonferroni correction (0.05/ $3=0.02$; level of significance $=0.02$ ) was used to allow for multiple comparisons. When comparing two time points during pregnancy, paired t-test was used to establish any changes. Changes in totalTC, holoTC, totalHC and holoHC between days 1 and 3 were also tested by the Hotellings T-square test throughout pregnancy and paired t-test within the respective gestation weeks. Intra-individual variation for the CobaSorb test was estimated by one-way analysis of variance for holoTC.

\section{Results}

\section{Participants}

We included 27 healthy Danish pregnant women. The mean age was 30, range 23-39 years, and parity was 1.6, range 
1-3. All women were on a normal diet and took folic acid supplementation at the time of inclusion.

\section{Cobalamin status}

Amongst the 27 participants, one displayed biochemical signs of an insufficient cobalamin status. Cobalamin was 114-126 pmol/L, MMA 0.24-0.52 $\mu \mathrm{mol} / \mathrm{L}$ and tHcy 5.6$9.4 \mu \mathrm{mol} / \mathrm{L}$. She did not differ from the other participants in any other way, and her data have been included in the study.

The cobalamin status during pregnancy is presented in Table 1 . The cobalamin levels declined gradually throughout pregnancy $(p<0.0001)$, while levels of MMA $(p=0.002)$ and tHcy $(\mathrm{p}<0.0001)$ increased (Table 1$)$. No overall change in holoTC was observed during pregnancy. However, when comparing weeks 24 and 36 a small increase was detected $(p=0.008)$. In contrast, levels of totalTC increased throughout pregnancy $(\mathrm{p}<0.0001)$ resulting in mean increases of $55 \mathrm{pmol} / \mathrm{L}$ (week 13 compared to week 24) and $130 \mathrm{pmol} / \mathrm{L}$ (week 13 compared to week 36). HoloHC levels decreased during pregnancy $(\mathrm{p}<0.0001)$ and the changes were caused by a decline in Cbl-HC $(\mathrm{p}<0.0001)$, while analog-HC remained constant (Table 1). A small decrease in totalHC was found when comparing weeks 13 and $36(p=0.007)$. Changes in Cbl-HC, analog-HC and apoHC are illustrated in Figure 1. In accordance with previous studies $(5,20,21)$, levels of hemoglobin declined during pregnancy, while creatinine displayed a small increase from week 24 to week 36. EMV showed a small increase during pregnancy (data not shown).

\section{Cobalamin absorption}

We examined the absorption of cobalamin three times during pregnancy in 27 women (Table 2). We judged that 21 women were able to absorb cobalamin based on the CobaSorb test. Upon loading with $\mathrm{CNCbl}$, the women increased more than $10 \mathrm{pmol} / \mathrm{L}$ and more than $22 \%$ in holoTC at all $(\mathrm{n}=19)$ or

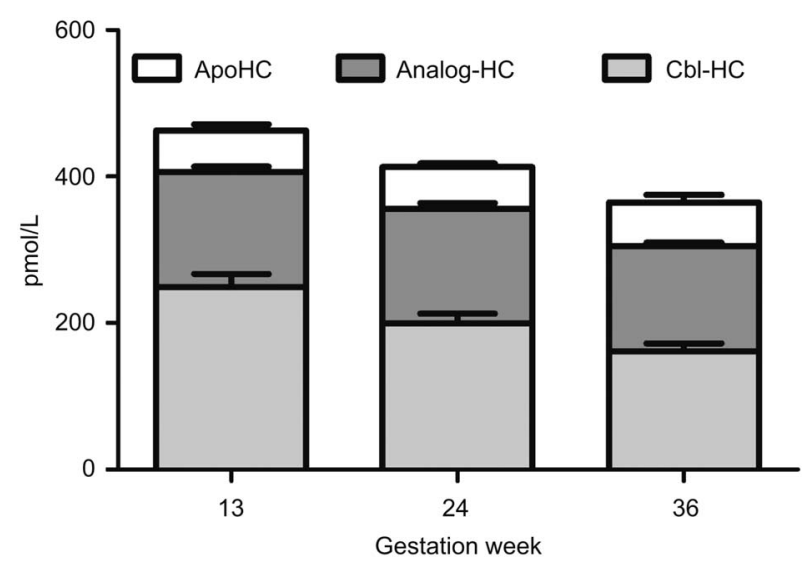

Figure 1 Changes in haptocorrin (HC) fractions during pregnancy. Means and SEM of various fractions of HC for 27 pregnant women analyzed at gestational week 13, -24 and -36 .

at two of the three $(n=2)$ examinations. In six women, baseline holoTC was above $65 \mathrm{pmol} / \mathrm{L}$ and upon loading with $\mathrm{CNCbl}$ the increase in holoTC was below $10 \mathrm{pmol} / \mathrm{L}$ for one or the other of the three examinations. We have previously shown that the CobaSorb test has limitations for baseline holoTC $>65 \mathrm{pmol} / \mathrm{L}$ (11). Because of that we reanalyzed all samples from the six participants by the C-CobaSorb (11). Judged by C-CobaSorb all six participants absorbed cobalamin, since upon loading with $\mathrm{CNCbl}$ they showed an increase in $\mathrm{CNCbl}$ of more than $6 \mathrm{pmol} / \mathrm{L}$ and more than $300 \%$ (Table 2).

Figure 2 shows the changes in holoTC and totalTC during pregnancy estimated by the CobaSorb test as alternations between baseline values and levels obtained after 2 days with three-times $9-\mu \mathrm{g}$ cobalamin intake. The increase in holoTC was similar throughout pregnancy and comparable to the increase observed for a previously published non-pregnant study group (10). Also, as shown in the non-pregnant study,

Table 1 Markers for cobalamin status during pregnancy.

\begin{tabular}{|c|c|c|c|c|c|}
\hline & Ref. Int. & Week 13 & Week 24 & Week 36 & $\begin{array}{l}\text { p-Value } \\
\text { Week 13-24-36 }\end{array}$ \\
\hline Cobalamin, pmol/L & $200-600^{\mathrm{a}}$ & $297^{\mathrm{g}}(126-475)$ & $244^{\mathrm{h}}(114-412)$ & $217^{\mathrm{i}}(114-357)$ & $<0.0001^{\mathrm{j}}$ \\
\hline HoloTC, pmol/L & $40-150^{\mathrm{b}}$ & $56(19-190)$ & $53^{\mathrm{h}}(20-91)$ & $60(21-116)$ & 0.03 \\
\hline TotalTC, pmol/L & $560-1550^{\mathrm{b}}$ & $884^{\mathrm{g}}(614-1265)$ & $952^{\mathrm{h}}(650-1240)$ & $1014^{\mathrm{i}}(714-1490)$ & $<0.0001^{\mathrm{j}}$ \\
\hline HoloHC, pmol/L & $190-590^{\mathrm{c}}$ & $338^{\mathrm{g}}(230-476)$ & $318^{\mathrm{h}}(218-500)$ & $292^{\mathrm{i}}(214-420)$ & $<0.0001^{\mathrm{j}}$ \\
\hline $\mathrm{Cbl}-\mathrm{HC}, \mathrm{pmol} / \mathrm{L}$ & $60-400^{\mathrm{d}}$ & $211^{\mathrm{g}}(100-368)$ & $194^{\mathrm{h}}(92-313)$ & $157^{\mathrm{i}}(88-248)$ & $<0.0001^{\mathrm{j}}$ \\
\hline Analog-HC, pmol/L & $100-380^{\mathrm{d}}$ & $155(77-243)$ & $154^{\mathrm{h}}(80-228)$ & $144^{\mathrm{i}}(87-194)$ & 0.06 \\
\hline TotalHC, pmol/L & $240-680^{\mathrm{c}}$ & $391(254-574)$ & $376(246-550)$ & $348^{\mathrm{i}}(232-532)$ & 0.03 \\
\hline $\mathrm{MMA}, \mu \mathrm{mol} / \mathrm{L}$ & $0.1-0.3^{\mathrm{e}}$ & $0.1(0.05-0.3)$ & $0.1^{\mathrm{h}}(0.06-0.5)$ & $0.16^{\mathrm{i}}(0.07-0.4)$ & $0.002^{\mathrm{j}}$ \\
\hline $\mathrm{tHcy}, \mathrm{mmol} / \mathrm{L}^{\mathrm{g}}$ & $4.5-7.9^{\mathrm{e}}$ & $3.8^{\mathrm{g}}(1.0-5.8)$ & $4.6(2.6-7.3)$ & $5.0^{\mathrm{i}}(2.5-10.4)$ & $<0.0001^{\mathrm{j}}$ \\
\hline Creatinine, $\mu \mathrm{mol} / \mathrm{L}$ & $50-90^{\mathrm{f}}$ & $45(36-56)$ & $44^{\mathrm{h}}(34-56)$ & $47(31-75)$ & 0.03 \\
\hline
\end{tabular}

Cobalamin, holoTC, totalTC, saturated HC (holoHC), cobalamin bound to HC (Cbl-HC), analog bound to HC (analog-HC), totalHC, plasma methylmalonic acid (MMA) and plasma homocysteine (tHcy) measured at gestation weeks 13, 24 and 36 for 27 healthy Danish women. Median and [range] are indicated. Reference interval for non-pregnant individuals are from ${ }^{\mathrm{a}} \mathrm{Nexo}(17),{ }^{\mathrm{b}} \mathrm{Nexo}$ et al. (13), ${ }^{\mathrm{c}} \mathrm{Morkbak}$ et al. (15), ${ }^{\mathrm{d}}$ Hardlei and Nexo (16), ${ }^{\mathrm{e}}$ Rasmussen et al. (18) and ${ }^{\mathrm{f}} \mathrm{Rustad}$ et al. (19). ${ }^{\mathrm{g}}$ Significant difference between weeks 13 and 24 estimated by

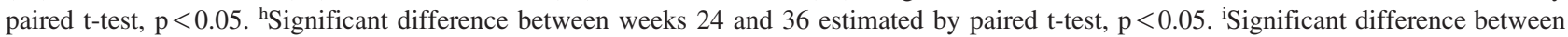

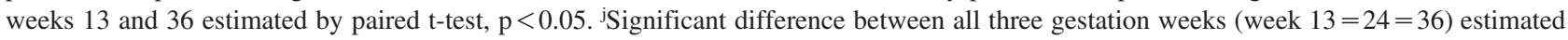
by Hotellings t-squared test with Bonferroni corrections, $\mathrm{p}<0.02$. 
Table 2 Changes in holoTC (CNCbl-TC) during the CobaSorb (C-CobaSorb) test in 27 pregnant women examined three times during pregnancy.

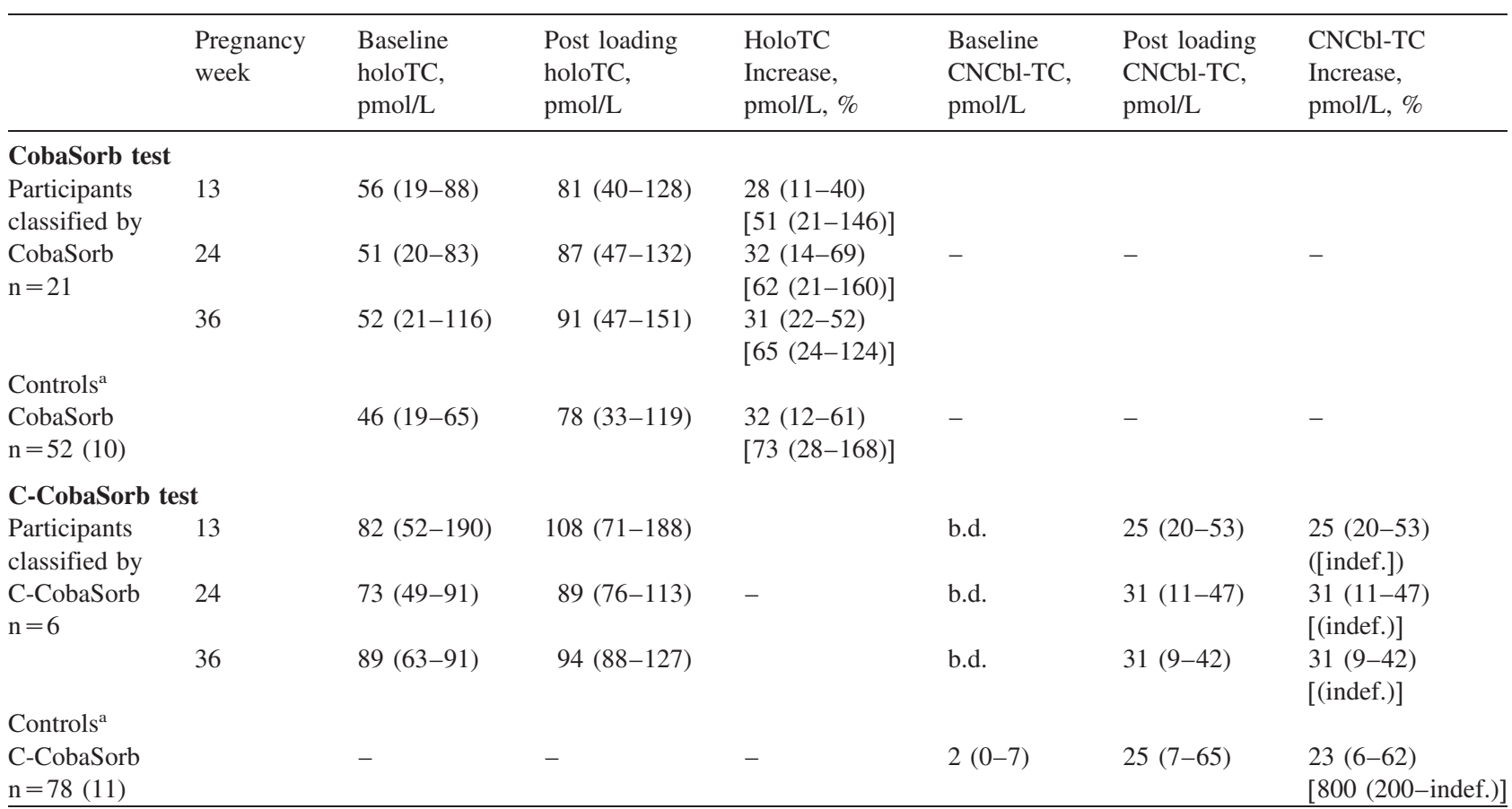

Blood samples were removed at baseline and after 2 days loading with three-times $9 \mu \mathrm{g}$ oral $\mathrm{CNCbl}$ (see Method sections for details). All samples from participants with one or more baseline holoTC $>65 \mathrm{pmol} / \mathrm{L}$ that increased $<10 \mathrm{pmol} / \mathrm{L}$ in holoTC upon loading with $\mathrm{CNCbl}$ at one or the other pregnancy week were further analyzed by the C-CobaSorb test (see Methods section for details). Median and [range]

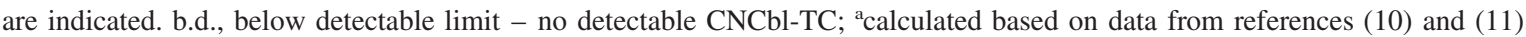

we observed an average decrease of $30-50 \mathrm{pmol} / \mathrm{L}$ in totalTC from day 1 to day 3 during the CobaSorb test at pregnancy weeks 13 and 24; however, a decrease of this magnitude was not observed in week $36(<5 \mathrm{pmol} / \mathrm{L})$ (Figure 2$)$.

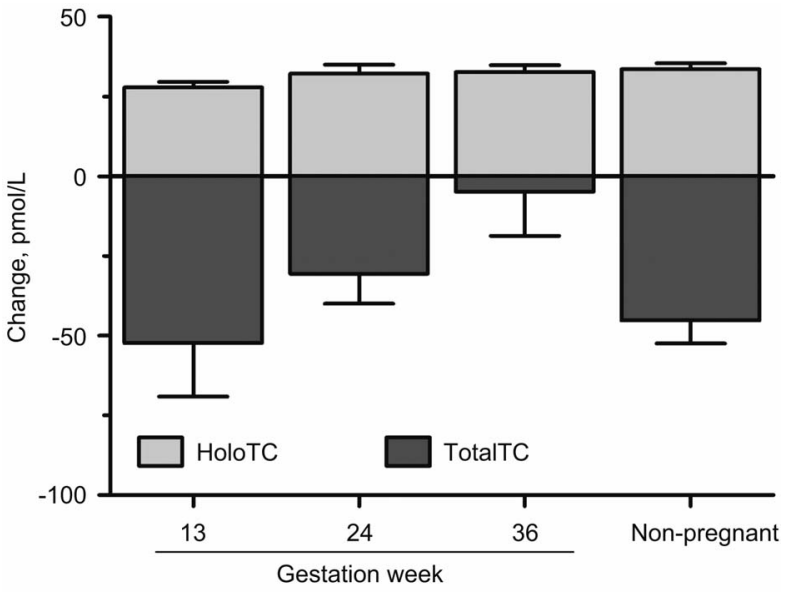

Figure 2 Cobalamin absorption during pregnancy. Alterations in holoTC and totalTC between baseline values and levels obtained after two days intake of 3 times $9 \mu \mathrm{g}$ of cobalamin. Mean and SEM are indicated for 27 pregnant women at gestational week 13, -24 and -36 (left part of the plot). Non-pregnant individuals classified by the CobaSorb test as cobalamin absorbers $(10)(n=52)$ were used for comparison (right part of the plot).
Based on the assumption that the absorption of cobalamin remains unchanged during pregnancy and by comparing the change in holoTC from day 1 to day 3 in all three gestation weeks, we find a Coefficient of Variance of $14 \%$ on the intraindividual variance of the CobaSorb test.

\section{Discussion}

Studies of cobalamin status in pregnant women often displayed a decreased level of plasma cobalamin particular in late pregnancy $(5,6,20)$ accompanied by a small but significant increase in the metabolic markers MMA and tHcy $(6,20)$. We wanted to further explore the cause for this alteration and we showed that the uptake of cobalamin as judged by the CobaSorb test remained unchanged during pregnancy.

All 27 pregnant women examined were found to absorb cobalamin with an increase in holoTC of the same magnitude as the non-pregnant individuals (10) (Figure 2) and no significant change in the ability to absorb cobalamin was found between gestation weeks 13, 24 and 36. Based on this, we conclude that uptake of cobalamin during pregnancy is comparable to that of non-pregnant individuals. This has two implications: first, the decline in plasma cobalamin during pregnancy is unlikely to be caused by malabsorption of the vitamin and, second, absorption of cobalamin can be judged by the same criteria as used for non-pregnant individuals (10). 
The decrease in totalTC during the CobaSorb test comparing gestational weeks 13 and 24 suggests a rapid removal of holoTC by the cells not followed by an equally rapid delivery of newly synthesized TC. The lack of decrease in totalTC at week 36 may reflect the increased synthetic rate of TC as mirrored in the increased level of totalTC seen in week 36.

So far, the intra-individual variation for the results of the CobaSorb tests has not been assessed. Our data show that the absorption of cobalamin remains unchanged during pregnancy and can therefore be used to calculate this value. By comparing the change in holoTC from day 1 to day 3 in all three gestation weeks, we found an intra-individual variance on the CobaSorb test of $14 \%$.

In accord with previous published results $(5,6,20)$, cobalamin displayed a significant gradual decline during pregnancy and in the third trimester more than $50 \%$ of the women demonstrated a decrease in cobalamin of $\geq 30 \%$ as compared to first trimester values. In accordance with others (6), we observed no decline in holoTC from the first to the third trimester; contrary, a small increase was seen between weeks 24 and 36. TotalTC increased markedly throughout pregnancy.

The decline in total cobalamin combined with steady levels of holoTC suggests that the change in cobalamin is caused by changes in the fraction of cobalamin bound to HC. To further scrutinize this point, we examined the concentration of $\mathrm{HC}$ and its subtractions. HC occurs in three fractions, one saturated with cobalamin (Cbl-HC), one saturated with cobalamin analogs (analog-HC) and one unsaturated (apoHC). Our study is the first to report a direct measure of $\mathrm{Cbl-HC}$ and analog-HC during pregnancy. The concentration of Cbl-HC declined highly significantly during pregnancy. A decline of almost the same magnitude - though less significant - was observed for totalHC, while small and insignificant alterations were observed for analog-HC and apoHC (Figure 1). The results are in accord with previous data that used calculated and not measured values for Cbl-HC (cobalamin minus holoTC) and analog-HC [holoHC minus (cobalamin minus holoTC)] (6). The results have two implications. First, the decline in cobalamin during pregnancy is caused by a decline in Cbl-HC that in part is likely to be caused by a decline in totalHC. Second, Cbl-HC declines while analog$\mathrm{HC}$ remain unchanged and within the reference range of nonpregnant individuals (16). At present, we have no explanation for the steady state level of analog-HC during pregnancy.

The decline in cobalamin during pregnancy has been a special concern since it is followed by an increase in the metabolic markers MMA and tHcy $(6,20)$, also observed in this study. However, the increase in these metabolic markers is small in absolute terms and was suggested to be caused by an increased metabolic rate and possibly by an increase in the serum creatinine levels rather than indicating a negative cobalamin status during pregnancy $(5,6,20)$.

In conclusion, cobalamin absorption does not change during pregnancy as judged by the CobaSorb test and the same criteria for absorption as used for non-pregnant individuals can be applied. The pregnancy-related decline in cobalamin can be accounted for by alternations in Cbl-HC, while no change was observed for the biological active holoTC. Curiously, the amount of analogs attached to $\mathrm{HC}$ remained unchanged during pregnancy.

\section{Acknowledgments}

The authors would like to thank Anna-Lisa Christensen and Jette Fisker, Department of Clinical Biochemistry, Aarhus University Hospital, Aarhus, for excellent technical assistance. This work was supported by grants from the Aase and Ejner Danielsen Foundation, the Danish Council for Independent Research/Medical Science, and the Lundbeck Foundation.

\section{Conflict of interest statement}

Authors' conflict of interest disclosure: The authors stated that there are no conflicts of interest regarding the publication of this article. Research support played no role in the study design; in the collection, analysis and interpretation of data; in the writing of the report; or in the decision to submit the report for publication.

Research funding: None declared.

Employment or leadership: None declared.

Honorarium: None declared.

\section{References}

1. Carmel R, Green R, Rosenblatt DS, Watkins D. Update on cobalamin, folate, and homocysteine. Hematology Am Soc Hematol Educ Program 2003;62-81.

2. Groenen PM, van Rooij AI, Peer PG, Gooskens RH, Zielhuis GA, Steegers-Theunissen RP. Marginal maternal vitamin B12 status increases the risk of offspring with spina bifida. Am J Obstet Gynecol 2004;191:11-7.

3. Afman LA, Van Der Put NM, Thomas CM, Trijbels JM, Blom HJ. Reduced vitamin B12 binding by transcobalamin II increases the risk of neural tube defects. QJM 2001;94:159-66.

4. Shojania AM. Folic acid and vitamin B12 deficiency in pregnancy and in the neonatal period. Clin Perinatol 1984;11:43359.

5. Koebnick C, Heins UA, Dagnelie PC, Wickramasinghe SN, Ratnayaka ID, Hothorn T, et al. Longitudinal concentration of vita$\min \mathrm{B}(12)$ and vitamin $\mathrm{B}(12)$-binding proteins during uncomplicated pregnancy. Clin Chem 2002;48:928-33.

6. Morkbak AL, Hvas AM, Milman N, Nexo E. Holotranscobalamin remains unchanged during pregnancy. Longitudinal changes of cobalamin and their binding proteins during pregnancy and postpartum. Haematologica 2007;92:1711-2.

7. Schilling RF. Intrinsic factor studies II. The effect of gastric juice on the urinary escretion of radioactivity after the oral administration of radioactive vitamin B12. J Lab Clin Med 1953;42: 860-6.

8. Bor MV, Nexo E, Hvas AM. Holo-transcobalamin concentration and transcobalamin saturation reflect recent vitamin B12 absorption better than does serum vitamin B12. Clin Chem 2004;50: 1043-9.

9. Bor MV, Cetin M, Aytac S, Altay C, Nexo E. Nonradioactive 
vitamin B12 absorption test evaluated in controls and in patients with inherited malabsorption of vitamin B12. Clin Chem 2005;51:2151-5.

10. Hvas AM, Morkbak AL, Nexo E. Plasma holotranscobalamin compared with plasma cobalamins for assessment of vitamin B12 absorption; optimization of a non-radioactive vitamin B12 absorption test (CobaSorb). Clin Chem Acta 2007;376:150-4.

11. Hardlei TF, Morkbak AL, Bor MV, Bailey LB, Hvas AM, Nexo E. Assessment of vitamin B(12) absorption based on the accumulation of orally administered cyanocobalamin on transcobalamin. Clin Chem 2010;56:432-6.

12. Nexo E, Christensen AL, Petersen TE, Fedosov SN. Measurement of transcobalamin by ELISA. Clin Chem 2000;46:16439.

13. Nexo E, Christensen AL, Hvas AM, Petersen TE, Fedosov SN. Quantification of holotranscobalamin, a marker of vitamin B12 deficiency. Clin Chem 2002;48:561-2.

14. Morkbak AL, Heimdal RM, Emmens K, Molloy A, Hvas AM, Schneede J, et al. Evaluation of the technical performance of novel holotranscobalamin (holoTC) assays in a multicenter European demonstration project. Clin Chem Lab Med 2005;43: 1058-64.

15. Morkbak AL, Pedersen JF, Nexo E. Glycosylation independent measurement of the cobalamin binding protein haptocorrin. Clin Chim Acta 2005;356:184-90.

16. Hardlei TF, Nexo E. A new principle for measurement of cobalamin and corrinoids, used for studies of cobalamin analogs on serum haptocorrin. Clin Chem 2009;55:1002-10.

17. Nexo E. Variation with age of reference values for P-cobalamins. Scand J Haematol 1983;30:430-2.

18. Rasmussen K, Moller J, Lyngbak M, Pedersen AM, Dybkjaer L. Age- and gender-specific reference intervals for total homocysteine and methylmalonic acid in plasma before and after vitamin supplementation. Clin Chem 1996;42:630-6.

19. Rustad P, Felding P, Franzson L, Kairisto V, Lahti A, Mårtensson A, et al. The Nordic Reference Interval Project 2000: recommended reference intervals for 25 common biochemical properties. Scand J Clin Lab Invest 2004;64:271-84.

20. Milman N, Byg KE, Bergholt T, Eriksen L, Hvas AM. Cobalamin status during normal pregnancy and postpartum: a longitudinal study comprising 406 Danish women. Eur J Haematol 2006;76:521-5.

21. Milman N, Bergholt T, Byg KE, Eriksen L, Hvas AM. Reference intervals for haematological variables during normal pregnancy and postpartum in 434 healthy Danish women. Eur J Haematol 2007;79:39-46. 